\title{
Failure of transient lower oesophageal sphincter relaxation in response to gastric distension in patients with achalasia: evidence for neural mediation of transient lower oesophageal sphincter relaxations
}

\author{
R H HOLLOWAY, J BRYANT WYMAN, AND J DENT \\ From the Department of Medicine, Flinders Medical Centre, Bedford Park, South Australia, and Section of \\ Gastroenterology, Marshfield Clinic, Marshfield, Wisconsin, USA
}

SUMmaRY Transient lower oesophageal sphincter relaxation (LOSR) is the major mechanism underlying gastro-oesophageal reflux. The mediation and control of LOSRs are incompletely understood but evidence suggests a neural inhibitory mechanism. In this study we have evaluated the effect of gastric distension on LOS function in 16 patients with untreated idiopathic achalasia and compared it with that in $\mathbf{1 0}$ healthy controls. With the subjects sitting, the stomach was distended with a liquid mixture that generated $750 \mathrm{ml} \mathrm{CO}$. Oesophageal $\mathrm{pH}$ and motility were monitored for 10 minutes before and after distension. In normal controls, gastric distension induced a four-fold increase in the rate of LOSRs and gas reflux episodes (as evidenced by oesophageal common cavities), whereas this response was absent in the achalasia patients. Basal LOS pressure did not change in either group. These findings are consistent with the notion that transient LOSRs induced by gastric distension are neurally mediated, probably by the same inhibitory nerves that govern swallow mediated LOS relaxation.

Over recent years, a substantial body of evidence has accumulated indicating that the major mechanism underlying gastro-oesophageal reflux (GOR) is transient lower oesophageal sphincter relaxation (LOSR). ${ }^{1-6}$ The mechanisms underlying LOSRs have yet to be completely defined. Gastric distension, however, is a potent stimulus ${ }^{6}$ and we have recently shown that LOSR is also the mechanism by which gas is vented from the stomach during belching induced by gaseous gastric distension. ${ }^{37}$ The rapid onset and brief time course of LOSRs, and their abolition by cervical vagal cooling, ${ }^{8}$ sleep, ${ }^{1}$ and general anaesthesia imply neural mediation. In idiopathic achalasia, available evidence indicates that the inhibitory neural pathways to the LOS are impaired or absent resulting in incomplete or absent LOS relaxation with swallowing. ${ }^{10-14}$ If these inhibitory

Address for correspondence: Dr Richard Holloway, Gastroenterology Unit, Royal Adelaide Hospital, North Terrace, Adelaide, South Australia, 5000.

Accepted for publication 27 October 1988. pathways also mediate LOSRs induced by gastric distension, this response would be expected to be absent in such patients. Our aim in this study was to evaluate the LOS response to gaseous gastric distension in patients with untreated idiopathic achalasia.

Methods

SUBJECTS

Studies were done on 16 patients (11 men, five women) aged from 16 to 80 years (mean 50), with untreated idiopathic achalasia. These patients were referred for investigation and treatment of dysphagia. Radiological examination uniformly showed absent peristalsis in the thoracic oesophagus, failure of the LOS segment to open normally, and varying degrees of oesophageal dilation. All patients had upper gastrointestinal endoscopy and in none was there evidence of tumour at the gastrooesophageal junction. Seven of the patients had retained food or secretions in the oesophagus. 
Oesophageal manometry showed absent peristalsis in the distal two-thirds or more of the oesophagus and absent or incomplete LOS relaxation to water swallows. Ten healthy asymptomatic volunteers (eight men, two women) aged 18 to 34 years (mean 23) served as controls. All subjects gave written informed consent and the protocol was approved by the Ethical Review Committees of the Flinders Medical Centre and the Marshfield Clinic.

OESOPHAGEAL MANOMETRY AND PH

RECORDINGS

Manometric recordings were made using an eight lumen assembly that incorporated a sleeve sensor at its distal end. The sleeve monitored LOS pressure. Side holes monitored pressure in the stomach, and in the oesophageal body at $3,7,11$, and $15 \mathrm{~cm}$ above the LOS. A side hole situated in the pharynx monitored swallows. The gastric, LOS, and oesophageal lumens were perfused with distilled water at $0.5 \mathrm{ml} / \mathrm{min}$ and the pharyngeal lumen at $0.13 \mathrm{ml} / \mathrm{min}$ by a pneumohydraulic capillary perfusion pump. Pressures were sensed by external transducers. Oesophageal $\mathrm{pH}$ was monitored with a miniature $\mathrm{pH}$ electrode positioned $5 \mathrm{~cm}$ above the LOS. The performance and calibration of the electrode were checked in buffers of $\mathrm{pH} 4$ and 7 before and after each experiment. Signals from the pressure transducers and $\mathrm{pH}$ meter were recorded on an eight channel chart recorder.

\section{PROTOCOL}

Subjects were studied after an overnight fast. No attempt was made to remove any food or secretions that may have been retained within the oesophagus. Before intubation, they were instructed how to distinguish between urge for and occurrence of belching (oesophago-pharyngeal gas reflux). After intubation, the subjects rested supine for 10 minutes to accommodate to the presence of the manometric assembly and $\mathrm{pH}$ electrode. They then sat up, with their legs over the side of the bed and feet resting on the floor. Spontaneous patterns of motility and $\mathrm{pH}$ were recorded in two consecutive 10 minute periods before and after gastric distension. At the end of each study the baselines of each manometric channel were determined with the perfusion running both with the catheter horizontal and hanging vertically. This procedure allowed for accurate comparison of pressures among different channels in the two body positions.

The stomach was distended with $750 \mathrm{ml} \mathrm{CO}$ generated intragastrically by a two component liquid mixture (Field's Negative C, Field Group Chemicals, Sydney, NSW). The first component was an alkali

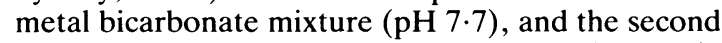
component a food grade acid solution ( $\mathrm{pH} 2 \cdot 0$ ). Fifteen millilitres of each component was instilled directly into the stomach through the central core of the manometric assembly to generate $750 \mathrm{ml} \mathrm{CO}$ within 10 seconds. After effervescence has stopped, the combined solutions had a $\mathrm{pH}$ of $3 \cdot 3$. Additionally, $2 \mathrm{ml}$ of a surfactant agent (Bubble-breaker, Field Group Chemicals) was added to the first component to minimise foaming.

DATA ANALYSIS

Tracings were analysed for evidence of gas GOR, for the motor events associated with gas GOR, and for the presence of transient LOSRs. Oesophageal common cavities were used as indicators of gas GOR. These were defined as abrupt simultaneous rises of basal oesophageal pressure to intragastric pressure in at least the lower two oesophageal recording sites. This pattern of pressure change has been identified previously in normal subjects and animals and accepted as a marker of gastrooesophageal gas flow. ${ }^{7516}$

The abrupt onset of these events served as the reference point for analysis of $\mathrm{pH}$ and motor events related to gas GOR. Oesophageal $\mathrm{pH}$ changes associated with each common cavity were determined by comparing $\mathrm{pH}$ values $5 \mathrm{~s}$ before and after the onset of the common cavity. The tracings were also scanned for the presence of transient LOSRs. These were defined as abrupt (within $5 \mathrm{~s}$ ) falls in LOS pressure to a level $4 \mathrm{mmHg}$ or less above intragastric pressure for at least $5 \mathrm{s.}^{2}$ End expiratory basal LOS pressure was referenced to intragastric pressure and derived as a one minute visual mean for each minute of the observation periods.

Statistical analysis was performed using the MannWhitney U-test for paired and unpaired values. A p value of $<0.05$ was accepted as indicating statistical significance. Data in the text are expressed as mean (SD) unless stated otherwise.

\section{Results}

NORMAL SUBJECTS

In the basal state, normal subjects exhibited a low rate of transient LOSRs and common cavities. Gaseous gastric distension induced a prompt and four-fold increase in the rate of both these events (Figs 1,2). The response was evident within the first minute after the onset of distension and was maintained throughout the distension period although $74 \%$ of the transient LOSRs and common cavities occurred within the first five minutes. The onset of all common cavities occurred during transient LOSRs. As well as increasing the rate of transient LOSRs, gastric distension also increased significantly the proportion of LOSRs associated with gas GOR from $34 \%$ to $94 \%\left(\chi^{2}=20 \cdot 5, p<0 \cdot 01\right)$. 


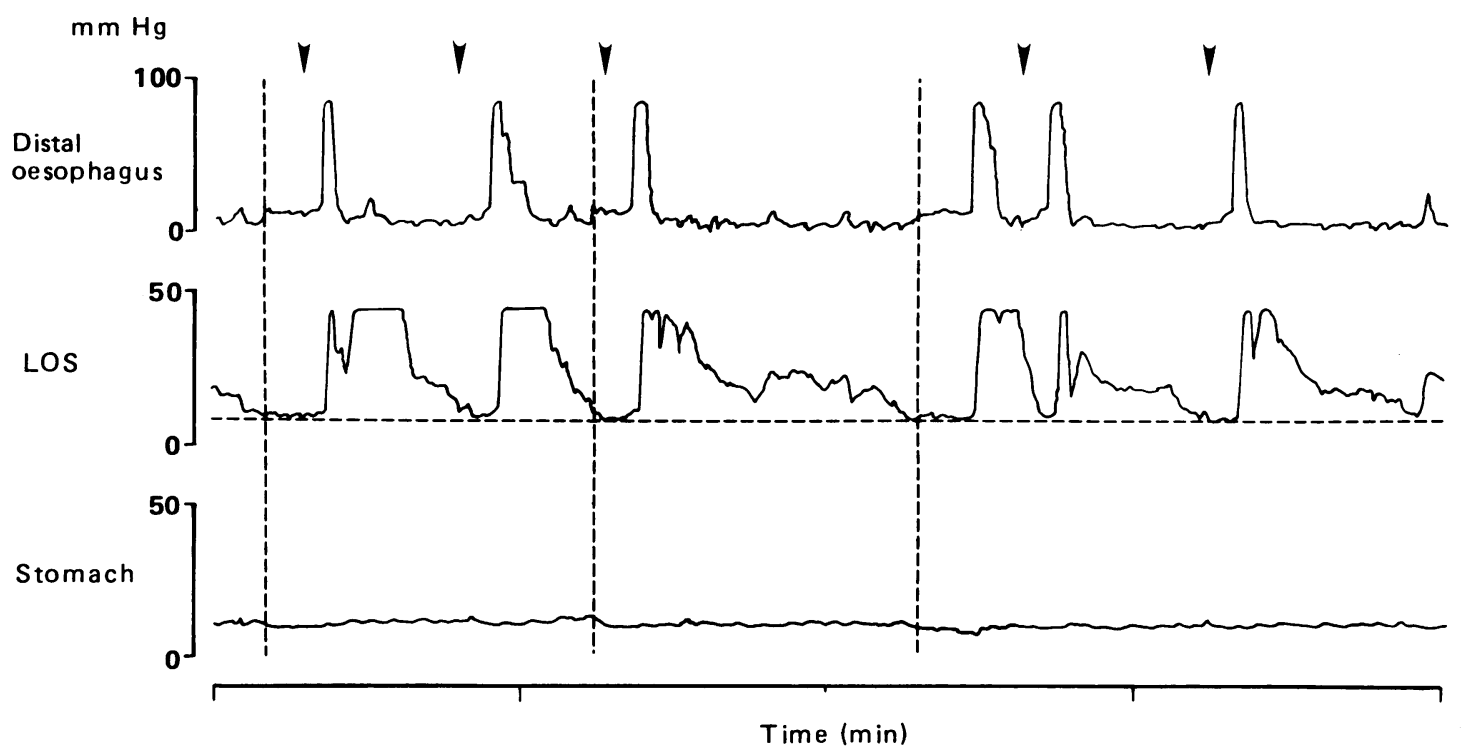

Fig. 1 Recording of oesophageal and gastric motility from the first four minutes after gaseous gastric distension with $750 \mathrm{ml}$ $\mathrm{CO}_{2}$ in a normal subject. The horizontal dashed line indicates intragastric pressure. The arrowheads indicate the timing of swallows. Three episodes of gas reflux as evidence by oesophageal common cavities (vertical dotted lines) have occurred during transient LOS relaxation. LOS - lower oesophageal sphincter.

The urge to belch was indicated on 10 occasions before distension and on 59 occasions afterwards. Belching was observed on two occasions, both after gastric distension. The majority $(83 \%)$ of belch urges occurred at the onset of or during a common cavity. Belch urge was always associated with the extension

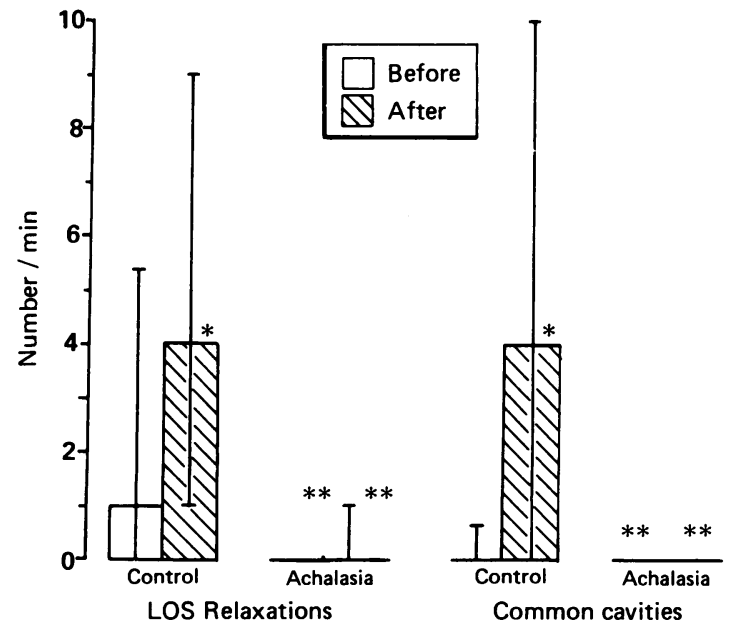

Fig. 2 Pooled data showing the effect of gaseous gastric distension on the rate of transient LOS relaxation and oesophageal common cavities. Data are expressed as median values (range). ${ }^{*} p<0.05$ v pre, ${ }^{* *} p<0.01 \mathrm{v}$ control. of the common cavity as far as the two most proximal oesophageal recording sites and was not experienced if the common cavity was limited to the distal two sites. Such limitation occurred only in the setting of gas GOR during a normal peristaltic sequence in which the oncoming peristaltic contraction prevented further upward flow of the refluxed gas. Resting oesophageal $\mathrm{pH}$ ranged between 5.2 and $6.8 \mathrm{pH}$ units. A small drop in oesophageal $\mathrm{pH}$ occurred with the majority $(83 \%)$ of the common cavities, the median change being $-0.5 \mathrm{pH}$ units (range $-2.6-$ $+0 \cdot 2 \mathrm{pH}$ units).

\section{ACHALASIA PATIENTS}

In sharp contrast to the response in normal subjects, only one transient LOSR occurred in the achalasia patients in the basal state and none during gastric distension (Figs 1, 3). No common cavities were observed. Belch urge was indicated by six of the patients on 75 occasions ( 24 before and 51 after distension) 51 of these occurring in only two patients. Belching occurred on 38 occasions (nine before and 30 after distension) one of these being associated with the single LOSR. Resting $\mathrm{pH}$ ranged between 4.5 and $6 \cdot 5$. No sudden changes in $\mathrm{pH}$ were observed.

BASA L LOS PRESSURE

Basal LOS pressure in the achalasia patients, 33.9 $(7 \cdot 5) \mathrm{mmHg}$ was significantly greater than that in the 

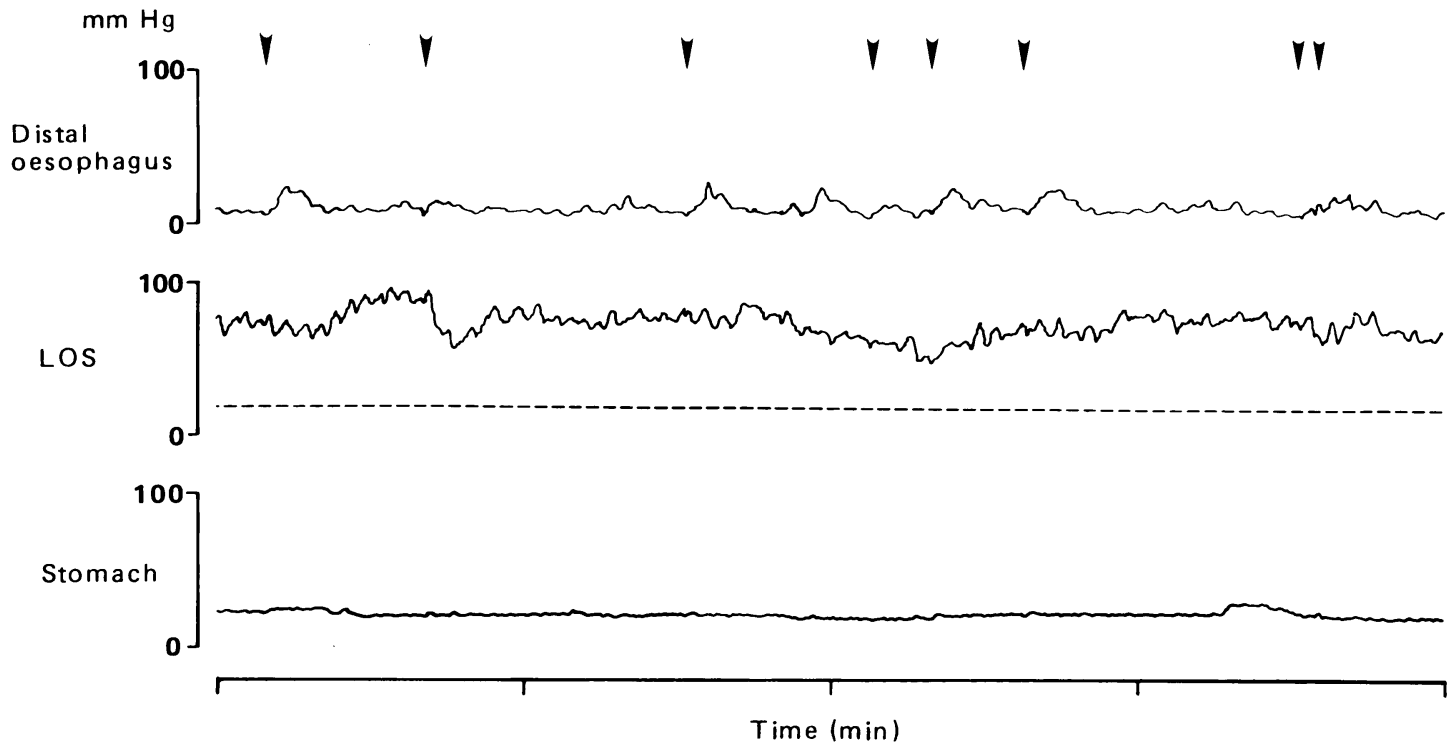

Fig. 3 Recording of oesophageal and gastric motility from the first four minutes after gaseous gastric distension with $750 \mathrm{ml}$ $\mathrm{CO}_{2}$ in an achalasia patient. The horizontal dashed line indicates intragastric pressure. The arrowheards indicate the timing of swallows. Neither LOS relaxations nor gas reflux (as evidenced by oesophágeal common cavities) have occurred. LOS-lower oesophageal sphincter.

normal subjects, $16 \cdot 0(5 \cdot 4) \mathrm{mmHg}(\mathrm{p}<0 \cdot 01)$, and did not alter significantly in either group after gastric distension. Modest and similar increases in intragastric pressure of between 2 to $5 \mathrm{mmHg}$ occurred after gastric distension in both normal subjects and the achalasia patients.

\section{Discussion}

In this study we examined the ability of gastric distension to induce transient LOSRs in patients with idiopathic achalasia. Such patients represent a human model of partial oesophageal denervation in which the major defect lies in the inhibitory innervation to the LOS. ${ }^{13 / 4}$ Our major finding was that achalasia patients, in sharp contrast with normal subjects, exhibited virtually no transient LOSRs basally and none in response to gastric distension.

Several lines of evidence suggest that LOSRs are neurally mediated. Transient LOSRs have an abrupt onset and brief time course, lasting from five to 30 seconds. ' Moreover, they are abolished by cooling of the cervical vagosympathetic trunks, ${ }^{x}$ and general anaesthesia." In normal subjects, gastric distension is a potent stimulus for LOSRs. ${ }^{\circ 7} \mathrm{We}$ have proposed that this response may be mediated by a vago-vagal reflex triggered by mechanoreceptors in the gastric wall. ${ }^{\circ}$ The virtual absence of transient LOSRs in achalasia patients is consistent with the impaired
LOS relaxation present in these patients and provides additional support for the notion that transient LOSRs are neurally mediated.

In normal subjects we observed a close association between belch urge and gas GOR sufficient to cause extension of the common cavity to the proximal oesophagus. This observation would suggest that belch urge results from distension of the proximal oesophageal body. Despite the absence of common cavities in the achalasia patients, some of the patients experienced belch urge. The reason for this is not clear. It is possible that common cavities may have been masked in some patients by increased intraoesophageal pressure. In addition, the oesophageal dilatation present in some could provide a greater capacitance to receive any refluxed gas thereby potentially reducing or blunting the abrupt rise in oesophageal pressure that characterises common cavities in normal subjects. The virtual lack of any transient LOSRs, however, provides strong supportive evidence for the failure of gas GOR. A recent study has shown that in the absence of straining, reflux occurs only when LOS pressure approaches undetectable levels. ${ }^{17}$ Except during the one transient LOSR, such pressures were never recorded in our achalasia patients. A more likely explanation for the experience of belch urge in some of the achalasia patients is that it results from the perception of oesophageal distension by swallowed air or secre- 
tions that have been retained in the oesophageal body. The belching observed in some patients most likely represents oesophagopharyngeal venting of this retained air.

A small drop in oesophageal $\mathrm{pH}$ occurred in association with gas reflux in the normal subjects. No such events were observed in the achalasic patients. Whilst it is possible that retained secretions or food in some patients may have prevented any $\mathrm{pH}$ change associated with gas reflux, ${ }^{18}$ the lack of any transient LOSRs or manometric correlates of gas reflux argues against any gas GOR having occurred undetected.

Previous observations on the neural defect in achalasia suggests two possible sites of interruption to the proposed neural reflex arc mediating LOSRs induced by gastric distension. More likely is disruption of the intrinsic inhibitory nerves mediating LOS relaxation in response to swallowing, these nerves possibly representing the final common pathway for LOS inhibition induced by several stimuli. The disruption, however, could be at the level of the vagal nerves. Athough conflicting, there is some evidence suggesting that the vagal innervation of the oesophagus and stomach is impaired in achalasia, ${ }^{19-21}$ This impairment could interrupt either the transmission of afferent impulses from the gastric mechanoreceptors to the brainstem, or the efferent supply to the intrinsic inhibitory nerves. Any demonstrable vagal damage, however, has only been partial or patchy and seem unlikely to account for the virtual absence of LOSRs in achalasia patients.

Some workers have pursued a mechanical approach to the question of the effect of gastric distension on LOS pressure and competence..$^{22-24} \mathrm{On}$ the basis of studies with in vitro models of sphincter function, they have proposed that gastric distension effaces the sphincter segment reducing its length and intrinsic pressure thereby producing incompetence. These models do not mimic adequately the physiologic properties of this region. First, the profile of 'gastric' pressure tested in these models does not resemble that seen in real life GOR. Second, the models do not take into account reflex LOS inhibition which is of paramount importance in normal and disordered transsphincteric flow. Such observations, made under highly artificial conditions, are not supported by our current observations nor previous findings. ${ }^{1}: 7 x$

In summary we have shown that patients with achalasia do not manifest transient LOSRs in response to gaseous gastric distension. Of broader importance is that this finding supports the notion that LOSRs triggered by gastric distension are neurally mediated. More detailed characterisation of the neural pathways involved may have important implications for the treatment of GOR disease.
Selective disruption of the neural mechanisms underlying LOSRs may allow for the control of pathological reflux whilst leaving normal LOS function intact.

Supported in part by grants from the National Health and Medical Research Council of Australia and from the Marshfield Medical Foundation. Dr R H Holloway was the recipient of a NH \& MRC Postgraduate Scholarship. The authors would like to thank Greg Skerven and Kathy Jackson for expert technical assistance and Jill Hanley for help in preparing the manuscript. This work was presented at the October 1986 meeting of the American Motility Society and published in abstracted form in Gastroenterology 1986; 91: 1055.

\section{References}

1 Dent J. Dodds WJ, Friedman RH, et al. Mechanism of gastroesophageal reflux in recumbent asymptomatic human subjects. J Clin Invest 1980; 65: 256-67.

2 Dodds WJ, Dent J, Hogan WJ, et al. Mechanisms of gastroesophageal reflux in patients with reflux esophagitis. N Engl J Med 1982; 307: 1547-52.

3 Patrikios J, Martin CJ, Dent J. Relationship of transient lower esophageal sphincter relaxation to post-prandial gastrocsophageal reflux and belching in dogs. Gastroenterology 1986; 90: 545-51.

4 Mittal RK. McCallum RW. Characteristics of transient lower esophageal sphincter relaxation in humans. Am J Physiol 1987; 252 (Gastrointest Liver Physiol 15): G636-41.

5 Dent J, Holloway RH, Dodds WJ, Toouli J. Mechanisms of lower oesophageal sphincter incompetence in gastro-oesophageal reflux discase. Gut 1988. (In press).

6 Holloway RH, Hongo M, Berger K. McCallum RW. Gastric distension: a mechanism for postprandial gastroesophageal reflux. Gastroenterology 1985; 89: 779-84.

7 Wyman JB, Dent J, Heddle R, et al. Belching: a clue to understanding of pathological gastroesophageal reflux [Abstract]. Gastroenterology 1984; 86: 1303.

8 Martin CJ, Patrikios J, Dent J. Abolition of gas reflux and transient lower esophageal sphincter relaxation by vagal blockade in the dog. Gastroenterology 1986; 91: $890-6$.

9 Cox MR, Martin CJ, Dent J, Westmore M. Effect of general anaesthesia on transient lower esophageal sphincter relaxation in the dog. Aust NZ J Surg 1988. (In press).

10 Rake GW. A case of annular muscular hypertrophy of the ocsophagus, (achalasia of the cardia without oesophageal dilatation). Guy's Hosp Rep 1926; 76: 14552.

11 Trounce JR, Denchar DC, Kauntze R, Thomas GA. Studies in achalasia of the cardia. QJ Med 1957; 26: 43343.

12 Adams CWM, Brain RHF, Trounce JR. Ganglion cells in achalasia of the cardia. Virchows Arch [Pathol Anat] 1976; 32: 75-9. 
13 Dodds WJ, Dent J, Hogan WJ, et al Paradoxical lower esophageal sphincter contraction induced by cholecystokinin - Octapeptide in patients with achalasia. Gastroenterology 1981; 80: 327-33.

14 Holloway RH, Dodds WJ, Helm JF, Hogan WJ, Dent J. Integrity of cholinergic innervation to the lower esophageal sphincter in achalasia. Gastroenterology 1986; 90: 924-9.

15 McNally EF, Kelly JE, Ingelfinger FJ. Mechanism of belching: effects of gastric distension with air. Gastroenterology 1964; 46: 254-9.

16 Stevens CE. Pressure events in bovine esophagus and reticulorumen associated with eructation, deglutition and regurgitation. Am J Physiol 1960; 199: 598-602.

17 Dent J, Dodds WJ, Hogan WJ, Toouli J. Factors that influence induction of gastroesophageal reflux in normal human subjects. Dig Dis Sci 1988; 33: 270-5.

18 Smart HL, Foster PW, Evans DF, Slevin B, Atkinson M. Twenty four hour oesophageal acidity in achalasia before and after pneumatic dilatation. Gut 1987; 28: 883-7.
19 Cassella RR, Brown AL, Sayre GP, Elliss FH. Achalasia of the esophagus: pathologic and etiologic considerations. Ann Surg 1964; 160: 474-87.

20 Woolam GL, Maher FT, Ellis FH. Vagal nerve function in achalasia of the esophagus. Surg Forum 1967; 18: 362-5.

21 Dooley CP, Taylor IL, Valenzuela JE. Impaired acid secretion and pancreatic polypeptide release in some patients with achalasia. Gastroenterology 1983; 84: 80913.

22 DeMeester TR, Wernly JA, Bryant GH, et al. Clinical and in-vitro analysis of determinants of gastroesophageal competence. Am J Surg 1979; 137: 39-48.

23 Petterson GB, Bombeck CT, Nyhus LM. The lower esophageal sphincter: mechanisms of opening and closure. Surgery 1980; 88: 307-14.

24 Bonavina L, Evander A, DeMeester TR, et al. Length of the distal esophageal sphincter and competency of the cardia. Am J Surg 1986; 151: 25-34. 